\title{
Transient Fuel-Film Compensation Strategy of PFI Gasoline Engine Based on Combined Film Model
}

\author{
Yongjun Huang, Guohua Chen \\ School of Energy and Power Engineering, Huazhong University of Scicence \& Technology, 430070 Wuhan, China
}

\begin{abstract}
For port-injected gasoline engines, an innovative strategy of transient fuel-film compensation is proposed to eliminate the impact of fuel-film dynamic effects and improve the transient air fuel ratio control accuracy. A combined fuel-film model composes of a long-term fuel-film and a short-term fuel-film was established. A transient fuel-film compensator model was analyzed in detail. The respective term of the fuel-film model is calculated by a share factor. The decay coefficients control the fuel compensation quantity and the active time. The validity of above models were verified though engine bench tests. The results show that this new strategy can effectively suppress the $\lambda$ offset during engine transient condition, the transient lambda deviation is less than $5 \%$ and stabilized within 1 second.
\end{abstract}

\section{Introduction}

PFI engine transient air-fuel ratio (AFR) accurate control is critical and difficult to achieve low emissions. Because during acceleration or deceleration, there are many factors that cause AFR deviation, as in [1], mainly including:

(1) The presence of dead time between actual load and measured load, as in [2]. The measured value can not truly reflect the actual value when the intake valve is closed.

(2) Intake lags. During accelerate process, since the actual intake air mass increases rapidly ,the calculated intake air mass is less than the actual intake air mass, causing a lean mixture; During decelerate process, since the actual intake air mass drops rapidly, the calculated intake air mass is greater than the actual intake air mass, resulting in a rich mixture, as in [3].

(3) Delay comes from sensor signal and processor computation, as in [4].

(4) Effect of fuel-film. General speaking, the evaporation and adhesion characteristics of fuel-film changes with pressure, as in [5]. In transition condition, changes of intake pressure causes fuel-film to change.

There are several existing solutions for fuel-film compensation: conventional PID controller, sliding mode controller, as in [6], neural network controller, as in [7], and model-based fuel-film compensator, as in [8]. Among them, the conventional PID controller is simple but too rough, its accuracy is limited. Neural networks and model-based controller usually involve complex mathematical theory using numerical integration for differential equations and a large number of matrix operations, as in [9].
This paper first proposes an application-oriented fuelfilm model and a transient fuel-film compensation algorithm, and then verifies the correctness of the proposed strategy by engine bench tests.

\section{Transient air fuel ratio control model}

\subsection{Non-dimensional pneumatic model}

To simplify the calculations, the basic fuel injection quantity is calculated using a non-dimensional pneumatic model. Engine load is represented by relative intake charge which is defined as the ratio of the actual intake air quantity of a cylinder per cycle under current pressure and temperature to the ideal capacity of a cylinder under standard condition.

According to the ideal gas equation:

$$
P V=M R T
$$

The actual air charge of cylinder can be expressed as:

$$
m_{c}=\frac{P_{c} V_{c}}{R T_{c}}
$$

The ideal intake charge of a cylinder under standard condition (pressure $P_{0}=101.3 \mathrm{KPa}$, temperature $T_{0}=0^{\circ} \mathrm{C}$ ) is:

$$
m_{s t d}=\frac{P_{0} V_{c}}{R T_{0}}
$$

According to the definition, the relative intake charge of cylinder is: 


$$
r 1=\frac{m_{c}}{m_{s t d}}=\frac{P T_{0}}{P_{0} T_{c}}
$$

At the intake valve closing (IVC), the intake manifold pressure and the in-cylinder pressure can be treated as balance, namely:

$$
P_{c}=P_{s}
$$

The ideal gas equation is used again and it gets:

$$
P_{c}=P_{i n}+P_{\text {int }}
$$

where, $V_{c}$ is cylinder volume, $m_{c}$ is in-cylinder air mass, $T_{c}$ is in-cylinder air temperature, $T_{0}$ is cylinder air capacity under standard condition, $P_{c}$ is in-cylinder air pressure , $P_{s}$ is intake manifold pressure, $P_{i n}$ is fresh air pressure portion in-cylinder, $P_{\text {int }}$ is residual gas pressure portion in-cylinder .

Therefore, the relative fresh air charge is described as:

$$
r 1_{i n}=\frac{P_{i n} T_{0}}{P_{0} T_{c}}=\frac{\left(P_{s}-P_{\mathrm{int}}\right) T_{0}}{P_{0} T_{c}}=\left(P_{s}-P_{\mathrm{int}}\right) f_{p s}
$$

As shown in 0 , the relative intake charge is linear to the intake manifold pressure.

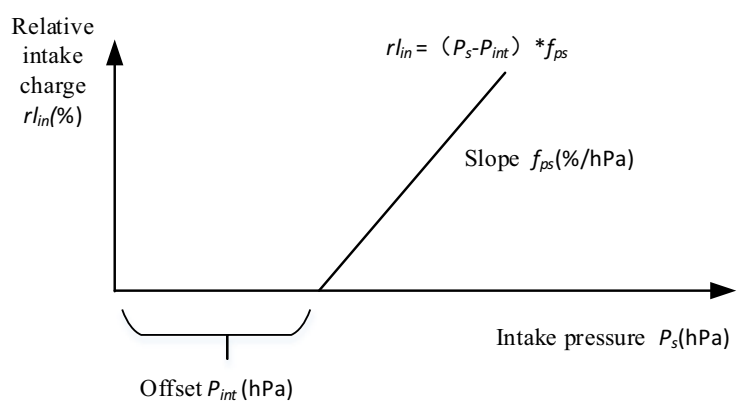

Figure 1. Relationship between relative the intake charge and intake pressure.

The slope of linear equation $f_{p s}$ is calculated from intake air temperature, the intercept $P_{\text {int }}$ is calibrated as function of engine speed and the valve overlap angle.

\subsection{Calculation of steady-state film quantity and dynamic film delta}

It has been pointed out that the steady fuel-film mass is influenced by various factors such as engine load, speed and temperature. In fact, during the film compensation calculation process, the delta between the fuel-film mass is more meaningful than the actual amount of fuel-film. When dynamically switching between two steady conditions, the same film delta value can be used in acceleration and deceleration.
Since the steady film amount can not be measured directly, it needs to be calibrated indirectly. After the engine heats up, to start with a speed of $1200 \mathrm{r} / \mathrm{min}$ and lambda $=1$, switch between different loads, adjust the basic fuel-film amount according to the changes of airfuel ratio, thereby change the film compensation amount correspondingly.

The calibration criteria is: During the process of changing the engine load, if the area of lean and rich part of lambda is severe asymmetry, it is considered as the film compensation is inadequate or excessive; If they are basically equal, the preset fuel-film is considered as the reasonable amount of steady fuel-film amount at the very operating point.

The adjustment idea is: If there is a large dilute peak at acceleration or a large peak at deceleration, it means that the fuel-film compensation is inadequate, so the amount of compensation should be increased; If there is a large rich peak at acceleration or a large lean peak at deceleration, it means that the film compensation is excessive, so the amount of compensation should be reduced; Keep tuning the fuel-film variables until the lambda peak value of acceleration and deceleration is basically symmetry. The characteristic curve of basic fuel-film quantity is shown in 0 .

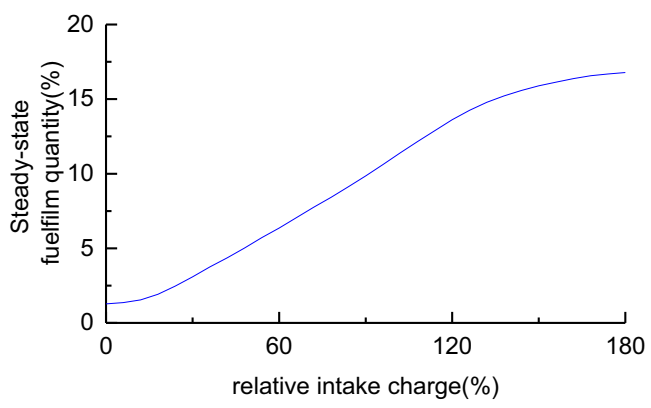

Figure 2. Characteristic curve of basic fuel-film quantity vs relative intake charge.

The basic amount of film is corrected according to the engine speed and coolant temperature. The correction coefficient is shown in 0 .

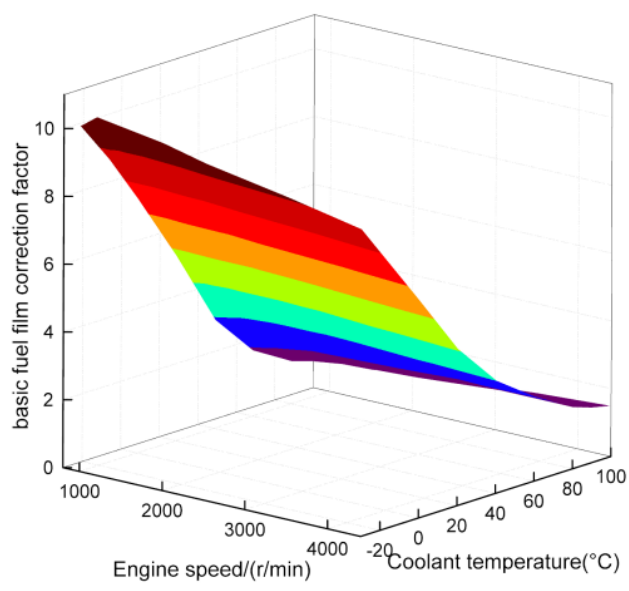

Figure 3. MAP of basic fuel-film correction factor. 


\subsection{Calculation of transient film compensation}

Nowadays, the widely used film model $\mathrm{x}-\tau$ model is derived based on physical model, as in [10]:

$$
\left\{\begin{array}{l}
\dot{m}_{f f}=x \dot{m}_{f i}-\frac{m_{f f}}{\tau} \\
\dot{m}_{f}=(1-x) \dot{m}_{f i}+\frac{m_{f i}}{\tau}
\end{array}\right.
$$

The $\mathrm{x}-\tau$ model is transformed to transfer function model using Laplace algorithm

$$
G(S)=\frac{1+(1-x) \tau S}{1+\tau S}
$$

In order to achieve the desired film compensation effect, the transfer function of film compensator should be an inverse function of the transfer function of film

$$
G(s)=G^{-1}(s)=\frac{1+\tau S}{1+(1-x) \tau S}
$$

where, $\dot{m}_{f i}$ is injection flow rate, $\dot{m}_{f f}$ is film variation rate, $\dot{m}_{f}$ is fuel flow rate into the cylinder, $x$ is deposit factor, $\tau$ is evaporation time constant, $s$ is Laplace operator.

According to the relationship between time-domain response and Laplace transform, making an inverse Laplace transform to the product of the film compensator transfer function and $1 / s$ will yield a time-domain representation of the step response of the film compensator

$$
G_{c}(t)=1+\frac{1+\tau X}{\tau-\tau X} e^{-\frac{t}{\tau-\tau X}}
$$

The above formula indicates that the ideal film compensation decays exponentially, the initial value and the decay rate are not only highly nonlinear but also timevarying. Since the exponential characteristic decays fast first then slow, the exponential decay film will be divided into two parts, a fast decay long-term film and a slow decay short-term film. They constitute a new combination film models.

The film model is shown in 0 . According to the knowledge of geometry, the area enclosed by the solid line and the axis is approximated to the sum of areas enclosed by dashed and dotted lines and the axes. Both long-term film and short-term film have positive compensation and negative compensation.

Features of the film model: long-term film is less in quantity but decays more slowly and lasts for a longer duration, it makes the transition of injection fuel much smoother, so it is helpful in stabilizing the air fuel ratio; Short-term film is larger in quantity but decays more faster and lasts for a shorter duration, it functions for compensating the severe mutation film at rapid changes in throttle, avoiding large air-fuel ratio pulse. Short-term film is extremely important for suppressing the air-fuel ratio peak. During acceleration, short-term film increases rapidly to the maximum value to avoid producing large lean peak .Because the acceleration process is completed in a very short time, the short term film should quickly decay, to avoid producing obvious rich peak. During deceleration, short-term film quickly reaches the minimum value to avoid producing larger rich peak then decays quickly to avoid producing obvious lean peaks.

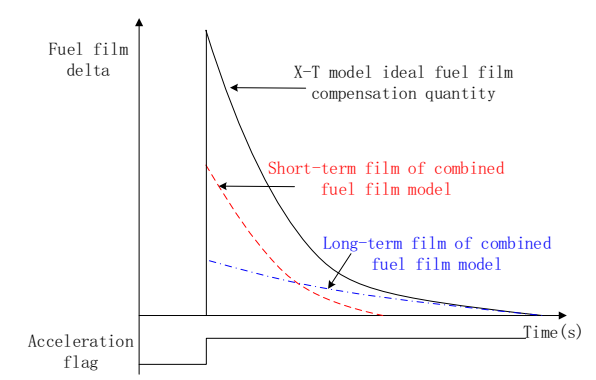

Figure 4. Combined fuel-film model.

The distribution of long-term film and short-term film is extremely important in derivation of film compensation. Upon the assignment of long-term film and short-term film of the film model, accurate calculations of compensation value for the film can be made. The longterm film and short-term film is decided based on different characteristics of engine (speed of film evaporation or adhesion). Because the film effects only affect the dynamic conditions, the steady conditions do not require compensation and the film compensation should be promptly ended after completion of dynamic behaviour. The overall scheme of transient film compensation is shown in 0 .

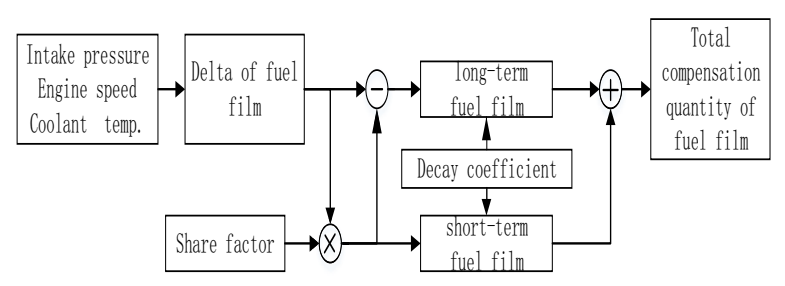

Figure 5. Overall transient compensation strategy.

\subsection{Calculation of share factor of long- term/short-term film}

The distribution ratio of long-term and short-term of the film is determined based on engine characteristics. The ratios of fuel-film distribution on acceleration and deceleration are different, and should be treated separately. The relative magnitude between long-term and short-term of the fuel-film varies with engine speed and temperature. Intake air pressure and air-flow mass are different at different engine speeds, resulting in different speeds of evaporation and adhesion of fuel-film. Wall temperature of Intake manifold is different at different engine coolant temperatures, resulting in different speeds of evaporation and adhesion of fuel-film too. The overall trend is that: as the speed increases, the film distribution 
coefficient increases; as the temperature rises, the film distribution coefficient increases too. The MAP of film distribution coefficients is shown in 0 and 0 .

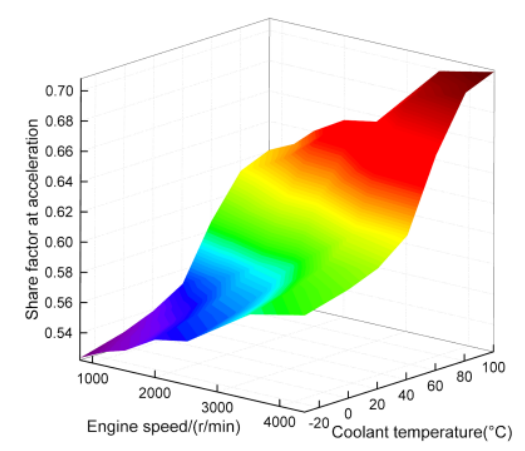

Figure 6. Share factor of fuel-film at acceleration.

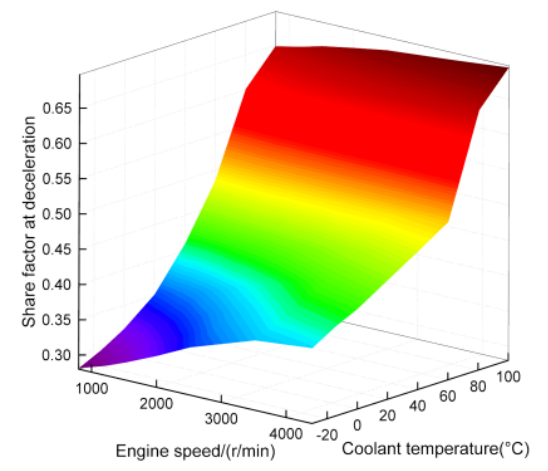

Figure 7. Share factor of fuel-film at deceleration.

\subsection{Calculation of decay factor of long- term/short-term film}

Upon finishing allocating the long-term film and shortterm film, the decay coefficient is used to control the decay rate and the corresponding duration of film compensation for acceleration and deceleration condition respectively. The bigger the decay coefficient is, the shorter the film lasts for; on the contrary, the smaller the decay coefficient is, the longer the film lasts for. The film decay coefficient is shown in 0 .

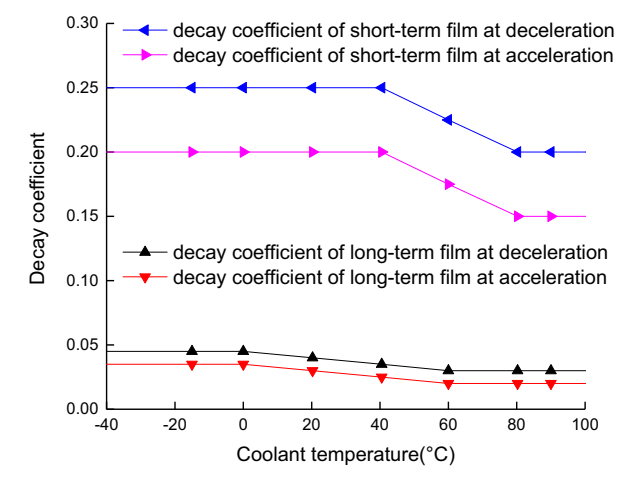

Figure 8. Decay coefficient of long-term/ short-term film.

On one hand, the decay coefficient mainly depends on the engine temperature. The respective decay coefficient of long-term film and short-term film varies in the same trend on both acceleration and deceleration working conditions. They decrease while temperature increases. Although the high temperature is conducive for the film to volatile, it seems that the decay coefficient should be increased. However, when the temperature is high, the basic film is usually small and the corresponding change of the magnitude of the film is small, hence the step size should be small.

On the other hand, the decay coefficient of both longterm film and short-term film is slightly bigger on deceleration than on acceleration. Since the intake manifold pressure decreases during deceleration, the film can evaporate faster than that on acceleration. The basic requirement is that the compensation value quickly decays when the film changes fast and the compensation value decays slowly when the film changes slowly. The film decay coefficient mainly affects the duration of the transient air-fuel ratio deviation. If the duration of deviation is too long, the film decay coefficient should be increased, to fasten the air-fuel ratio to recovery to normal value.

\subsection{Calculation of total film compensation}

The compensation quantities of long-term film and shortterm film are calculated in the same way, and the way they are calculated are similar for acceleration and deceleration. If an injection occurs between the current calculation and the last calculation, it means that the compensation fuel previously calculated has entered into the cylinder, so the last compensation fuel should be subtracted off. If no injection occurs (e.g. the first cycle at recovery from fuel cut-off), it means that the fuel-film compensation of previously calculation is not performed, so the last compensation fuel can not be subtracted, but accumulated. Furthermore, during the calculation of compensation of the short-term film, a dead-zone function is added to filter the compensation value. When the compensation amount is less than the threshold, no compensation would be used, to prevent unnecessary compensation to result in frequency fluctuations in mixture when the change of engine load is too small. The calculation model of fuel-film compensation is shown in 0 and 0 respectively.

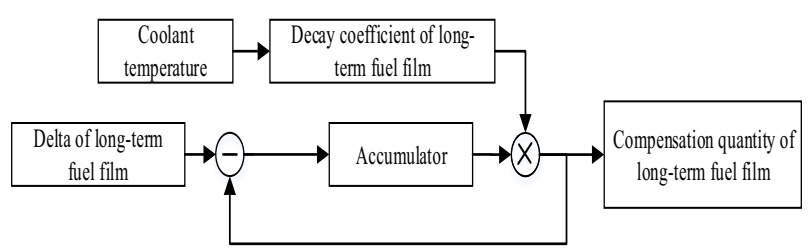

Figure 9. Long-term fuel-film compensation quantity computation module.

Both on acceleration and deceleration, the compensation value of long-term film $m_{l}(i)$ is calculated as:

$m_{l}(i)= \begin{cases}m_{f i}(i) \times f_{l x}, & i=1 \\ \sum\left[m_{f i}(i)-m_{l}(i-1)\right] \times f_{l x}, & i>1\end{cases}$ 
where, $m_{f l}(i)$ is the delta of long-term film at current calculation time, $x=a, d, f_{l a}$ is decay factor of long-term film for acceleration, $f_{l d}$ is decay factor of long-term film for deceleration.

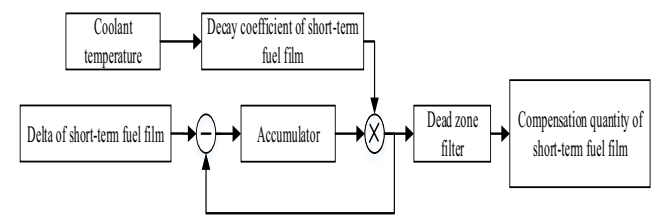

Figure 10. Short-term fuel-film compensation quantity computation module.

Both at acceleration and deceleration, the compensation value of short-term film $m_{s}(i)$ is calculated as:

$$
\begin{gathered}
m_{s}^{\prime}(i)= \begin{cases}m_{f s}(i) \times f_{s x}, & i=1 ; \\
\sum\left[m_{f s}(i)-m_{s}(i-1)\right] \times f_{s x}, & i>1\end{cases} \\
m_{s}(i)= \begin{cases}m_{s}^{\prime}(i), & \left|m_{s}^{\prime}(i)\right| \geq U ; \\
0, & \left|m_{s}^{\prime}(i)\right| \leq U\end{cases}
\end{gathered}
$$

where, $m_{f s}(i)$ is the delta of short-term film at current calculation time, $x=a, d, f_{s a}$ is decay factor of short-term film for acceleration, $f_{s d}$ is decay factor of short-term film for deceleration, $U$ is filter threshold.

\section{Experimental set-up}

The tests were conducted on a $1.5 \mathrm{~L}$ four-cylinder engine DFMA15 of Dengfeng Company. An overview of the engine bench configuration is shown in 0 and the engine specifications are listed in Table 1. Other devices included a bench control system F2000 of Xiangyi Power Instrument Company, an air-fuel ratio meter ES631, an exhaust analyzer LA4 and a CAN communication module ES581 of ETAS Company. The controller hardware is based on Freescale 32-bit processor MPC5634M. The advance software ASCET is used for graphically modelling and generating executable $\mathrm{C}$ code automatically. ES631 is installed upstream catalytic converter. Data are collected every 10ms using INCA7.0.

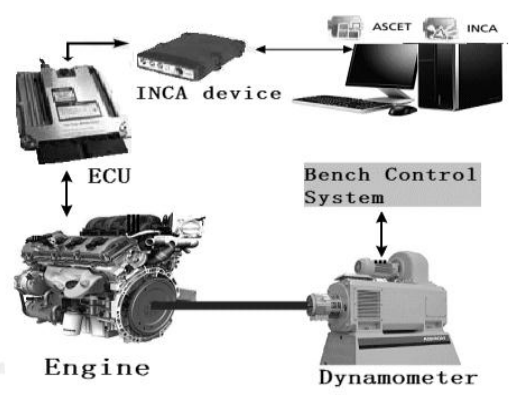

Figure 11. Sketch of engine bench configuration.
Table 1. Engine parameters.

\begin{tabular}{|l|l|}
\hline Bore/Stroke & $75 \mathrm{~mm} / 84 \mathrm{~mm}$ \\
\hline Compression ratio & 11 \\
\hline Displacement & $1.5 \mathrm{~L}$ \\
\hline Maximum torque & $145 \mathrm{~N} \mathrm{~m}($ at $4200 \mathrm{r} / \mathrm{min})$ \\
\hline Rated power & $85 \mathrm{~kW}$ (at $6000 \mathrm{r} / \mathrm{min})$ \\
\hline Fuel injection system & Port Fuel Injection \\
\hline Intake type & Normally Aspirated \\
\hline Number of cylinder & 4 \\
\hline Fire orders & $1-3-4-2$ \\
\hline
\end{tabular}

\section{Bench test results and analysis}

Since throttle tips in and tips out are typical transient conditions of engine, a rapid step of the electronic throttle is applied. In order to verify the validity of proposed fuelfilm model and the control model, the new compensation algorithm is compared to noncompensation algorithm and Delphy compensation algorithm. The test results are shown in 0, 0, 0 and 05 .

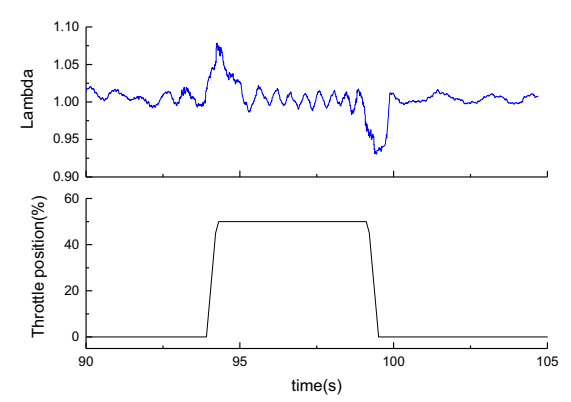

Figure 12. Throttle position varies $0-50 \%$, non-compensation.

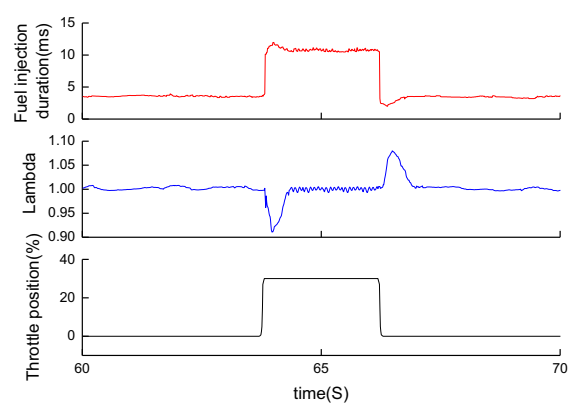

Figure 13. Throttle position varies $0-30 \%$, film compensation of Delphy is applied.

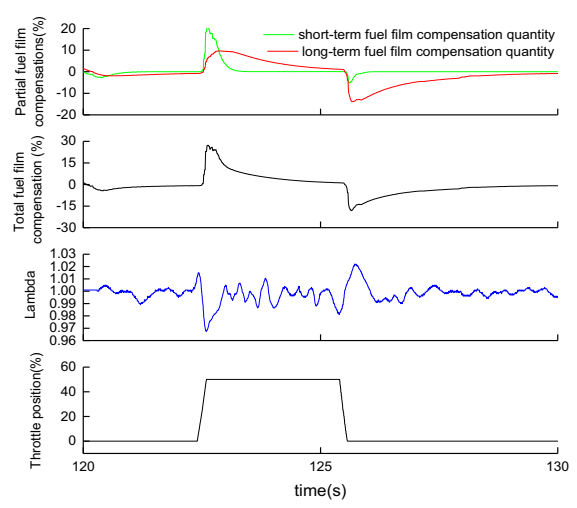

Figure 14. Throttle position varies $0-50 \%$, combination film compensation is applied. 


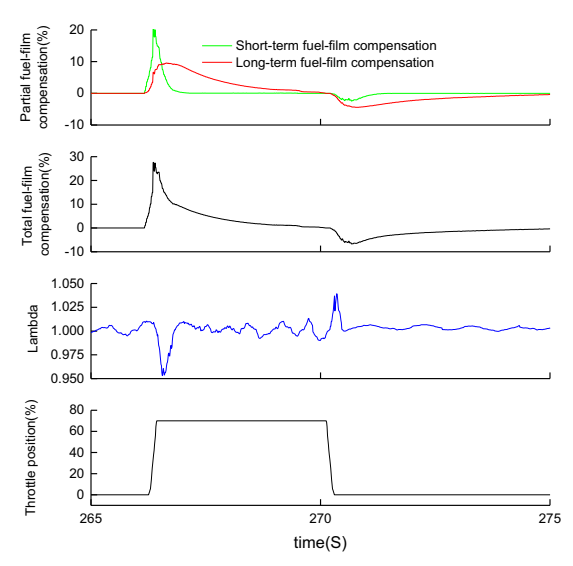

Figure 15. Throttle position varies $0-50 \%$, combination film compensation is applied.

\subsection{Comparison between combination film compensation algorithm and non-compensation algorithm}

In 0 , when no film compensation is used, the mixture is lean when acceleration and rich when deceleration, with a maximum deviation of about $8 \%$. In 0 and 0 , when the combination film compensation strategy is adopted, the mixture was enriched at acceleration and diluted at deceleration. The transient Lambda peak deviation is greatly reduced. The maximum deviation dropped to less than $5 \%$ and settling time is less than 1 second.

\subsection{Analysis of control effect of the combination film compensation algorithm at tip-in/tip-out}

As seen from the 0 and 0 ,when a tip-in occurs, both the long-term film and short-term film increases at the same time as the throttle opening increases until the throttle opening unchanged. The short-term film decays to zero rapidly, while the long-term film decays to zero slowly. The total compensation value first increases then decreases. When a tip-out occurs, the film compensation works in opposite direction. Both the long-term film and the short-term film decrease at the same time as the throttle turns smaller until the throttle opening unchanged. The short-term film decays to zero rapidly, while the long-term film decays to zero slowly. The total compensation value first increases then decreases. The total compensation value first reaches a minimum value then changes towards zero in an opposite direction.

The lambda is less than 1 and lasts for a short period of time at tip-in, it means that the acceleration has been enriched; The lambda is larger than 1 and lasts for a short period of time at tip-out, it means that the deceleration has been diluted; The lambda convergences to 1 rapidly after the throttle gets steady, it is controlled with the range from 0.99 to 1.01 .

\subsection{Comparison between combination film compensation algorithm vs other algorithm}

According to the comparison of 0,0 and 0 , the new compensation algorithm on transient air fuel ratio control reached the same level as the existing mature system.

\section{Conclusions}

As can be seen from the dynamic experiment results, at the same operating conditions, lambda excursion decreased obviously when the combination film compensation algorithm is used. The change of fuel-film on transient working condition can be controlled correctly and precisely by the fuel-film compensation control strategy. The maximum deviation of transient lambda is less than $5 \%$.

Transient On-line correction of the transition compensation in the event of coking on the valves, variations of individual engines, varying fuel quality etc will be investigated in future work. The transition compensation is corrected by means of an amplification factor which works on all parts of the transition compensation if considerable deviations in the air-fuel ratio are detected during load changes.

\section{References}

1. M. Takazawa, K. Komura, T. Kitamura, $S A E$ technical paper (2015)

2. Y. Yildiz, A. Annaswamy, D. Yanakiev, Control Engineering Practice, 18, 1369-1378 (2010)

3. C. Wang, J. Zhang, C. Yin, Chinese Journal of Mechanical Engineering, 27, 2 348-357 (2014)

4. R. Maurya, A. Agarwal, SAE technical paper (2011)

5. T. Takiyama, SAE Technical paper (2014)

6. M. Ichiyanagi, T. Suzuki, SAE technical paper (2015)

7. H. Toshitani, T. Shen, and J. Zhang, SAE technical paper (2011)

8. A. Gaeta, S. Santini, L. Glielmo, SAE technical paper (2003)

9. L. Wu, J. J. Liu, Applied Mechanics \& Materials, 643, 66-71 (2014)

10. Y. Zhao, T. Shen, X. Jiao, SAE technical paper (2013) 